Pacific Journal of Mathematics

HOMOLOGY COBORDISMS OF 3-MANIFOLDS, KNOT
CONCORDANCES, AND PRIME KNOTS 


\title{
HOMOLOGY COBORDISMS OF 3-MANIFOLDS, KNOT CONCORDANCES, AND PRIME KNOTS
}

\section{Charles LivingstoN}

\begin{abstract}
There is a close relation between link concordances and homology cobordisms of 3 -manifolds. Using this relationship we will prove that every closed, orientable 3-manifold is homology cobordant to an irreducible 3-manifold. Essentially the same construction will be used to prove that every knot in $S^{3}$ is concordant to a prime knot.
\end{abstract}

Kirby and Lickorish [3] have previously obtained the second result using a different construction. This answers problem 13 in Gordon [2].

Results of Schubert [6] show that the knots constructed are prime. In the second half of this paper we will generalize his results to characterize prime "generalized" cable knots. If $K_{1}$ is a knot in $S^{1} \times B^{2}$ and $K_{2}$ is a knot in $S^{3}$, we can form the $K_{1}$ cable of $K_{2}$ by mapping $S^{1} \times B^{2}$ into $S^{3}$, with $S^{1} \times\{0\}$ going to $K_{2}$. We will show that, with an obvious restriction, the $K_{1}$ cable of $K_{2}$ is prime if and only if $K_{1}$ is prime in $S^{1} \times B^{2}$. The final section gives a large set of examples of prime knots in $S^{1} \times B^{2}$.

I would like to thank Rob Kirby for his helpful suggestions and conversations.

1. Definitions and conventions. In all that follows manifolds and maps will be smooth and orientable. Intersections of submanifolds and of submanifolds with the boundary of a manifold will always be assumed to be transverse.

A knot $K$ in $S^{3}$ is an embedded $S^{1}$ in $S^{3}$, a link of $n$ components, $L$, is a collection on $n$ disjoint knots. By $N(K)$ we indicate a tubular neighborhood of $K$ in $S^{3}$. The meridian of a knot $K$ is any embedded $S^{1}$ in $\partial N(K)$ which bounds a $B^{2}$ in $N(K)$, and which is nontrivial in $H_{1}(\partial N(K) ; Z)$. A longitude of $K$ is an embedded $S^{1}$ in $\partial N(K)$ which is nontrivial in $H_{1}(\partial N(K) ; Z)$ but represents 0 in $H_{1}\left(S^{3}-\operatorname{int}(N(K)) ; Z\right)$.

Two links, $L_{1}$ and $L_{2}$, each of $n$ components are called concordant if there is an embedding $\bar{L}$ of $n$ disjoint copies of $S^{1} \times I$ into $S^{3} \times I$, with $\bar{L}\left(n\left(S^{1} \times I\right)\right) \cap S^{3} \times\{0\}=L_{1}$ and $\bar{L}\left(n\left(S^{1} \times\{I\}\right)\right) \cap S^{3} \times\{1\}=L_{2}$. Two 3-manifolds, $M_{1}$ and $M_{2}$, are homology cobordant if there is a 4-manifold $W$, with $\partial W=M_{1} \cup M_{2}$ and the map of $H_{*}\left(M_{i} ; Z\right) \rightarrow$ $H_{*}(W ; Z)$ an isomorphism.

A 3-manifold $M$ is irreducible if every embedded $S^{2}$ in $M$ bounds an embedded $B^{3}$. A link $L$ is irreducible, or unsplittable, if $S^{3}-L$ 
is irreducible.

2. Preliminary results. In order to construct homology cobordisms we must use the relationship between knot concordances and homology cobordisms. Proposition 2.1 below describes that relationship. We will then develop the essential tool for showing that a 3-manifold is irreducible, Proposition 2.3.

Given a $\operatorname{link} L$ in $S^{3}$ of $n$ components, and $n$ diffeomorphisms, $h_{\imath}$, of $T^{2}$ to itself, there is a natural way of forming a 3-manifold: remove $N(L)$ from $S^{3}$ and replace it according to the $h_{i}$ 's. In what follows we will be concerned only with integer surgery, in which case each $h_{i}$ maps the longitude to a meridian, and the meridian to a curve which is equivalent in $H_{1}(\partial N(K) ; Z)$ to a longitude plus $k$ meridians. If a manifold $M^{3}$ is obtained from surgery on a link $L_{1}$, and $L_{1}$ is concordant to $L_{2}$, there is a natural way to construct a homology cobordism between $M^{3}$ and $M^{3 *}$, where $M^{3 *}$ is obtained by surgery on $L_{2}$. That is, remove a tubular neighborhood of the concordance, and replace it according to the maps $h_{i} \times \mathrm{id}$, mapping $S^{1} \times S^{1} \times I$ to itself. This construction for knots is described in detail by Gordon in [1]. The following is a generalization to links of the theorem stated there.

Proposition 2.1. If a manifold $W$ is obtained by surgering a link concordance in $S^{3} \times I$, then it is a homology cobordism between its boundary components, $M_{1}$ and $M_{2}$.

Proof. Using Alexander duality it follows that the homology of the complement of the concordance is naturally isomorphic to the homology of the complement of $L$ in $S^{3}$. Then there are natural maps joining the Meyer-Vietoris sequence of $M_{i}$ to that of $W$, with almost all maps being isomorphisms. The proof is completed by applying the five lemma to these sequences.

A 3-manifold $M$ is said to have incompressible boundary if any embedded $S^{1}$ in $\partial M$ which bounds an embedded disk in $M$ also bounds a disk in $\partial M$. This is equivalent, via Dehn's lemma, to the usual algebraic definition.

LEMma 2.2. The complement of an open regular neighborhood of an irreducible link of more than one component in $S^{3}$ has an incompressible boundary. If the link has one component which is knotted this result also holds.

Proof. If for some component $K_{i}$ of $L$ we have some non- 
trivial $S^{1}$ in $\partial\left(N\left(K_{i}\right)\right)$ bounding an embedded disk $D$ in $S^{3}-L$, we have immediately that $K_{i}$ is unknotted. Further, $\partial\left(N\left(K_{i}\right) \cup N(D)\right)=$ $S^{2}$, and $K_{i}$ is the only component of $L$ in one of the components of $S^{3}$ bounded by $S^{2}$. The result readily follows.

Proposition 2.3. Let $K_{i}, i=1, \cdots, n$, be a collection of nontrivial knots in $S^{3}$, and $L$ a link of $n$ components in $S^{3}$ which is irreducible. Assume that $M=\left(S^{3}-\operatorname{int}(N(L))\right) \bigcup_{h_{1}}\left(S^{3}-\operatorname{int}\left(N\left(K_{1}\right)\right)\right) \bigcup_{h_{2}} \cdots$ $\bigcup_{h_{1}}\left(S^{3}-\operatorname{int}\left(N\left(K_{n}\right)\right)\right)$ with the $h_{i}$ diffeomorphisms. Then $M$ is irreducible.

Proof. Let $S$ be an embedded 2-sphere in $M$. We first show that $S$ can be isotoped to an embedding such $S \cap \partial(N(L))=\varnothing$ : If $S \cap \partial(N(L))$ is nonempty, take an innermost circle of intersection on $S$. That circle bounds a disk in either $S^{3}-N(L)$ or some $S^{3}-N\left(K_{i}\right)$. In either case, by Lemma 2.2 it bounds a disk on the boundary of that space. These two disks form a 2 -sphere in $S^{3}$, which bounds an embedded 3-ball. That 3-ball can now be used to isotop $S$ to eliminate at least one circle of intersection. By repeating this process we eventually have $S \cap \partial(N(L))=\varnothing$. We now have $S$ in one of the original $S^{3}$ 's. Now, perhaps using that $L$ is irreducible, we have that $S$ bounds a $B^{3}$.

3. Main construction. Our construction proceeds in two steps. We first find a description of a given manifold $M$ as surgery on a link of a given type. A certain concordance is performed which, by Proposition 2.1 gives a homology cobordism. Proposition 2.3 will imply that one end of that cobordism is irreducible.

Lemma 3.1. Any 3-manifold can be obtained by integer surgery on an irreducible link.

Proof. By a result of Lickorish [4] $M^{3}$ is obtained by integer surgery on some link. We will now alter that description. (See Rolfson [5] for a description of these alterations.) Grab a strand from each component of $L$ as indicated in Fig. 1 and add a +1
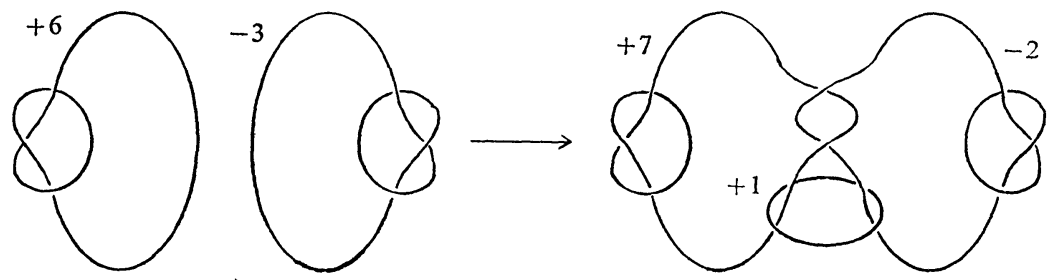

FIGURE 1 
linking each one. Each component of $L$ is now geometrically linked to the +1 . It is now immediate that the new link is irreducible.

Fig. 2 indicates a concordance of 2 knots in $S^{1} \times B^{2}$. This concordance will be essential in this section and in proving that every knot is concordant to a prime knot.
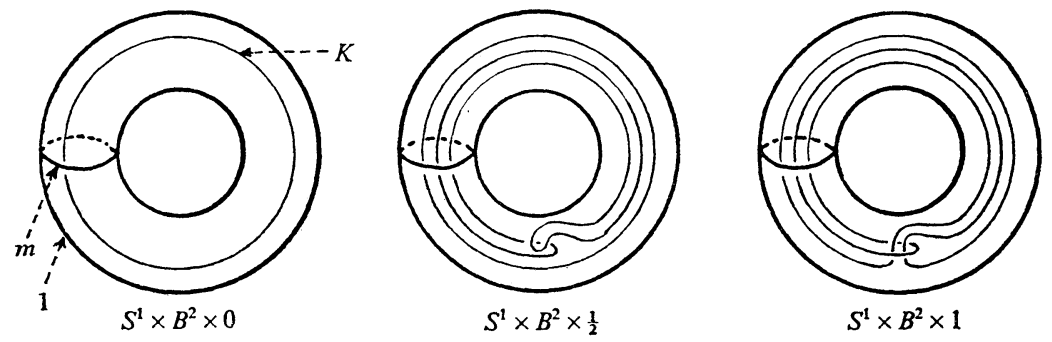

FIGURE 2

The concordance we perform on the link $L$ describing $M^{3}$ is just the concordance illustrated in Fig. 2 performed on each component. That is, for each component $K_{i}$ of $L$, we map $S^{1} \times B^{2}$ to $N\left(K_{i}\right)$ with $S^{1} \times\{0\}$ going to $K_{i}$, and similarly map $S^{1} \times B^{2} \times I$ to $N\left(K_{i} \times I\right) \subset$ $S^{3} \times I$. We can now map the concordance over also. For each of these maps there are actually many possible choices, depending on the image of the longitude 1 of $S^{1} \times B^{2}$. In each case pick the map so that the framing on $K_{i}$ corresponds to -1 surgery on the knot in $S^{1} \times B^{2}$, relative to the longitude indicated in Fig. 2. That is, there is a map as indicated in Fig. 3, preserving meridians.

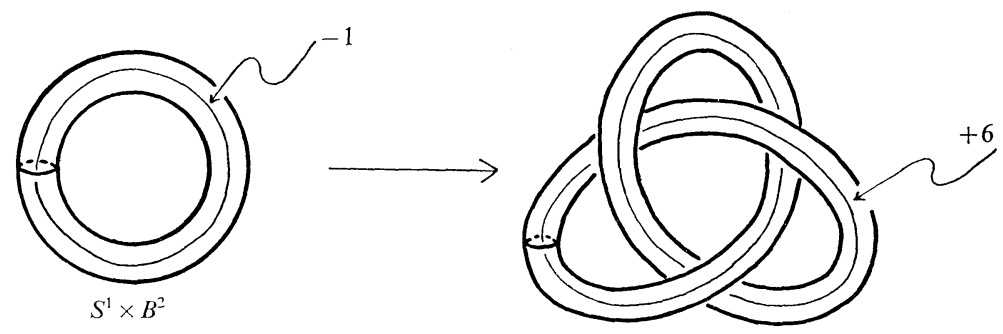

FIGURE 3

THEOREM 3.2. Every 3-manifold is homology cobordant to an. irreducible 3-manifold.

Proof. Describe $M^{3}$ as surgery on an irreducible link. Construct the concordance described above. This gives, using Proposition 2.1, a homology cobordism. The final manifold, $M^{3 *}$ is the union of the complement of an irreducible link, and the manifold shown in Fig. 4a. That manifold is the complement of the circle $C$. drawn in Fig. 4b. Redrawing the picture, and then removing the -1 shows that 
this manifold is a knot complement. See Fig. 4c and 4d. Hence, applying Proposition 2.3 gives the desired result.
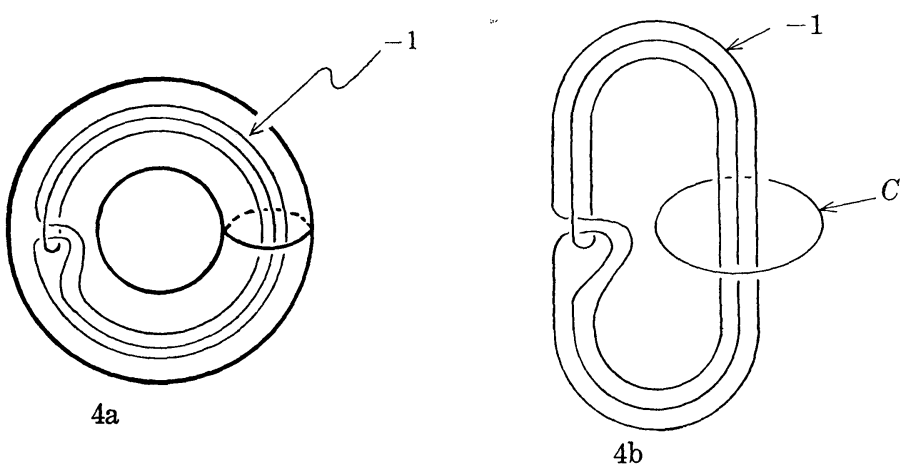

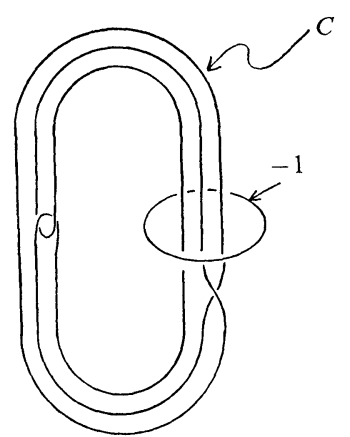

$4 \mathrm{c}$

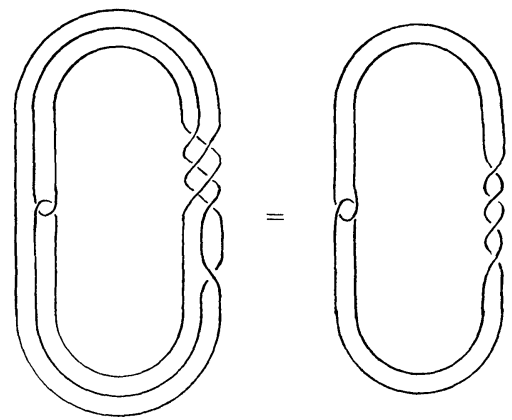

$4 d$

FiguRe 4

REMARK. Akbulut has informed me of a proof that integral surgery on any composite knot gives an irreducible 3-manifold. A similar argument to his, along with Proposition 2.3, gives the result that integral surgery on an irreducible link with all components knots gives an irreducible manifold. Hence another proof of Theorem 3.2 can be given, by changing the concordance used above to one taking each component to a composite knot. It is interesting to note that in the proof given earlier, all components finish as prime knots, as will be proven later.

Akbulut's construction is as follows. Let $K_{1}$ and $K_{2}$ be knots in $S^{3}$. Form a 4-manifold $W$ by attaching one copy of $B^{4}$ to another, identifying $N\left(K_{1}\right)$ in the first copy to $N\left(K_{2}\right)$ in the second. (The choice of attaching maps will determine the framing of the final knot.) The boundary of this manifold is clearly the union of 2 knot complements and is hence irreducible. However, this 4-manifold is the same one as is obtained by surgery on $K_{1} \# K_{2}$. This can be seen by "sliding" the cone point of $K_{2}$ in the second $B^{4}$ into the first. See Fig. 5 . 

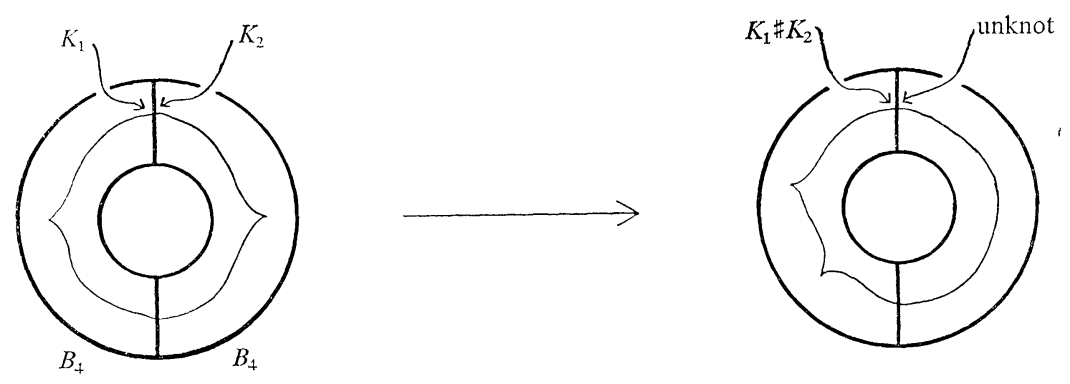

FIGURE 5

Another way of seeing this is to construct $W$ by first identifying the 2 copies of $B^{4}$ along 2 copies of $B^{3}$ in $N\left(K_{1}\right)$ and $N\left(K_{2}\right)$. This new manifold is just $B^{4}$. The identification is completed by adding a 2-handle to $B^{4}$ along $K_{1} \# K_{2}$. See Fig. 6 .
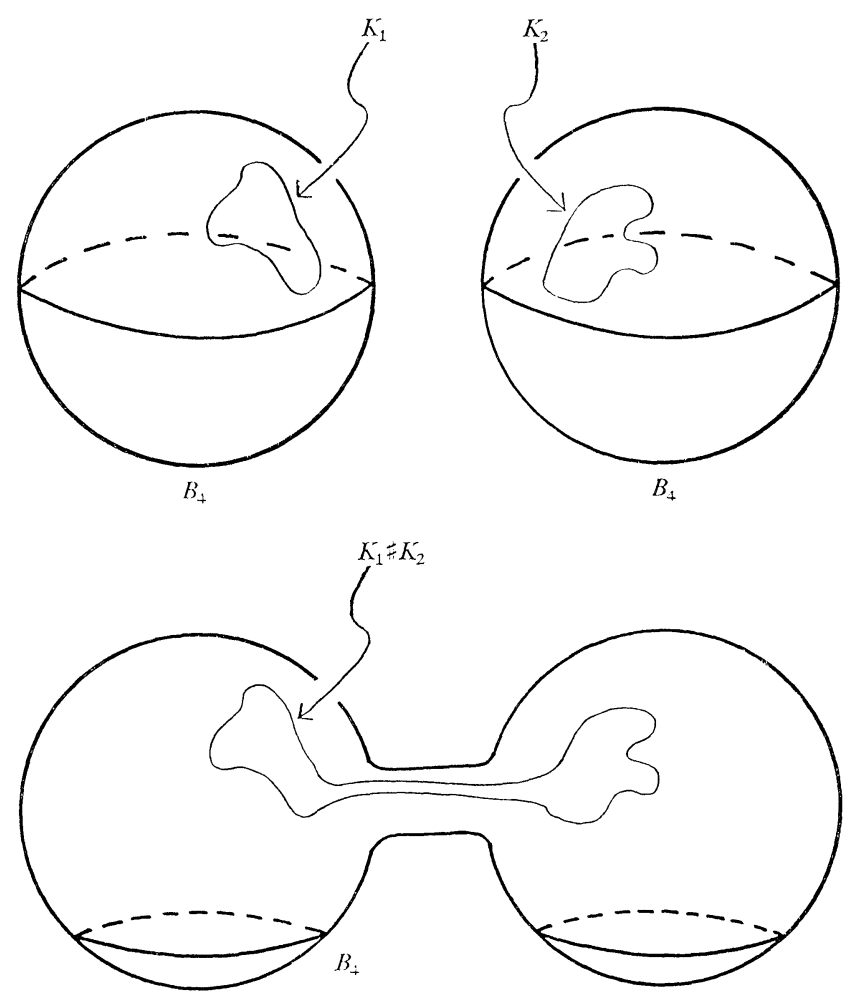

FIgURE 6

The same technique illustrated in Fig. 4 can be used to show that -1 surgery on the knot in $S^{1} \times B^{2}$ illustrated in Fig. 7a gives the complement of the knot $C$ in Fig. 7b. Hence all the 3-manifolds obtained by surgery on the knot $K_{1}$ in Fig. 7c and -1 surgery on any knot $J_{n}$ in Fig. $7 \mathrm{~d}$ is the union of the complement of the knot $C$ with a second copy of itself. 


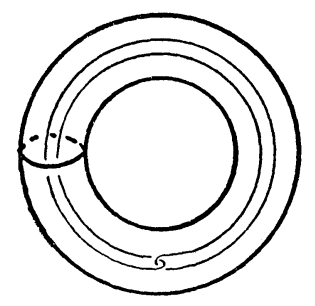

7 a.

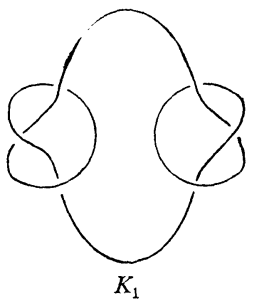

$7 c$.

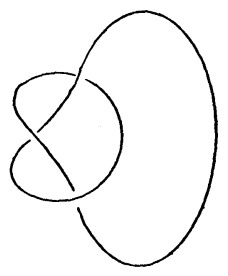

$7 \mathrm{~b}$.

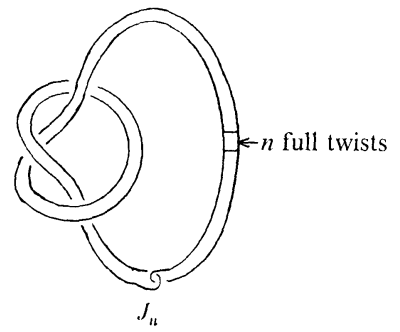

$7 d$.

FIGURe 7

4. Generalized cable knots. Before proving our main theorem concerning prime knots, we will set up some notation. By a splitting$S^{2}, S$, for a knot $K$ (in $S^{3}$ or $S^{1} \times B^{2}$ ) we indicate an embedded 2 -sphere, $S$, intersecting $K$ in exactly 2 points. A knot in either $S^{3}$ or $S^{1} \times B^{2}$ is prime if for every splitting- $S^{2}, S, S$ bounds some 3-ball, $B$, with $(B, B \cap K)$ a trivial pair. See Fig. 8. The winding number of a knot $K$ in $S^{1} \times B^{2}$ is that element $z$ of $H_{1}\left(S^{1} \times B^{2} ; Z\right)$ with $z \geqq 0$ and $K$ representing $z$. The wrapping number of a $K$ is the minimum number of intersections of $K$ with a disk $D$ in $S^{1} \times B^{2}$ with $\partial D=$ meridian. See Fig. 9. If $K_{1}$ is a knot in $S^{1} \times B^{2}$ and $K_{2}$ is a knot in $S^{3}$, the $K_{1}$ cable of $K_{2}$ is the knot in $S^{3}$ formed by mapping $S^{1} \times B^{2}$ into $N\left(K_{2}\right)$, and considering the image of $K_{1}$ under this map. The only restriction on the map of $S^{1} \times B^{2}$ into $N\left(K_{2}\right)$ is that it maps a meridian to a meridian. See Fig. 10.
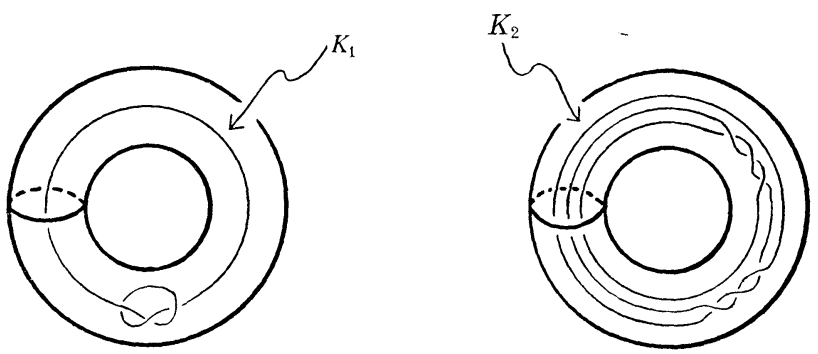

$K_{1}$ is prime in $S^{3}$, but not on $S^{1} \times B^{2}$.

$K_{2}$ is prime in $S^{1} \times B^{2}$, but not in $S^{3}$. (See $\S 5$ ).

FIGURE 8 


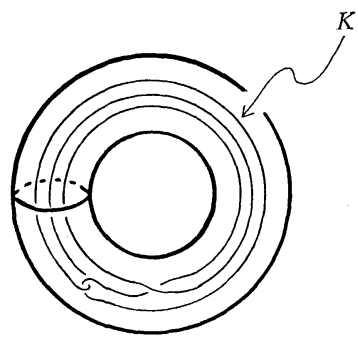

winding number $K=1$

wrapping number $K=3$

(See §5)

FiguRe 9
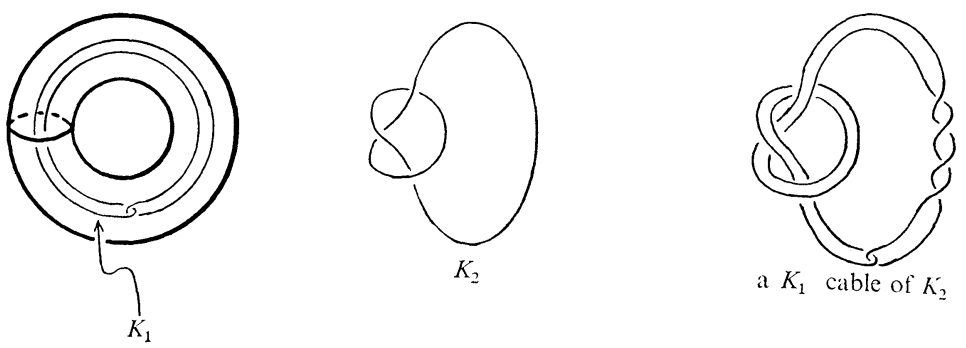

FIgURe 10

LEMMA 4.1. If $K_{1}$ is a knot in $S^{1} \times B^{2}$ with wrapping number $K_{1} \geqq 1$, and $K_{2}$ is any nontrivial knot in $S^{3}$, the $K_{1}$ cable of $K_{2}$ is nontrivial in $S^{3}$.

Proof. If the $K_{1}$ cable of $K_{2}, K$, is trivial, then the longitude of $K$ bounds an embedded disk, $D$, in $S^{3}-K$. We will show first that $D \cap \partial N\left(K_{2}\right)$ can be made empty. If $D \cap \partial N\left(K_{2}\right)$ is nontrivial, let $S^{1}$ be an innermost circle, on $D$, of intersection. $S^{1}$ bounds an embedded disk, either in $S^{3}-N\left(K_{2}\right)$ or $S^{1} \times B^{2}-K_{1}$. Using either the fact that $K_{2}$ is nontrivial or that wrapping number $K_{1} \geqq 1$, we have that $S^{1}$ is trivial on $\partial N\left(K_{2}\right)$. Hence the disk it bounds on $\partial N\left(K_{2}\right)$ along with the one it bounds on $D$ form an $S^{2}$. That $S^{2}$ bounds a $B^{3}$ which can be used to isotop $D$ to eliminate a circle of intersection. Hence, proceeding in this manner we eventually have $D \cap \partial N\left(K_{2}\right)=$ $\varnothing$, so $D$ is contained in $S^{1} \times B^{2}$. But this would imply that wrapping number $K_{1}$ is 0 , contradicting the hypothesis.

THEOREM 4.2. Let $K_{1}$ be knot in $S^{1} \times B^{2}$ with wrapping number $>$ 1 , and let $K_{2}$ be any nontrivial knot in $S^{3}$. The $K_{1}$ cable of $K_{2}$ is prime if and only if $K_{1}$ is prime in $S^{1} \times B^{2}$.

Proof. If $K_{1}$ is not prime in $S^{1} \times B^{2}$, then $K_{1}$ is obtained from a knot $K_{1}^{\prime}$ in $S^{1} \times B^{2}$ by connect summing. $K_{1}^{\prime}$ has wrapping number $>$ 1 , as any isotopy of $K_{1}^{\prime}$ in $S^{1} \times B^{2}$ could be extended to a neighborhood of $K_{1}^{\prime}$, and then to $K_{1}^{\prime}$ connect sum any knot. We then have 
that the $K_{1}$ cable of $K_{2}$ is the connect sum of some nontrivial knot and the $K_{1}^{\prime}$ cable of $K_{2}$, which, by Lemma 4.1 is nontrivial.

To prove the converse, assume that $S$ is a splitting 2 -sphere for the $K_{1}$ cable of $K_{2}$. We will show that there is an isotopy such that $S \cap \partial N\left(K_{2}\right)=\varnothing$. Then we will have $S \subset S^{1} \times B^{2}$. The primeness of $K_{1}$ will then imply that the $B^{3}$ bounded by $S$ in $S^{1} \times B^{2}$ satisfies $\left(B^{3}, B^{3} \cap K\right)=$ trivial pair.

Denote by $\underline{A} S$ with small open neighborhoods of $S \cap K$ removed. $A \cap \partial N\left(K_{2}\right)$ consists of a collection of circles. If any such circle is null homologous on $A$, let $S^{1}$ denote an innermost one. $S^{1}$ bounds an embedded disk in either $S^{3}-N\left(K_{2}\right)$ or $S^{1} \times B^{2}-N\left(K_{2}\right)$. In either case we get $S^{1}$ is null homologous on $\partial\left(N\left(K_{2}\right)\right)$. $\left(K_{2}\right.$ is knotted and wrapping number $K_{1} \geqq 1$.) The disk bounded by $S$ on $\partial\left(N\left(K_{2}\right)\right)$ along with the one it bounds on $A$ form an embedded $S^{2}$ in $S^{3}$, which bounds a $B^{3}$ in $S^{3}$. This $B^{3}$ can be used to isotop $S$ to eliminate (at least) one circle of intersection. See Fig. 11.

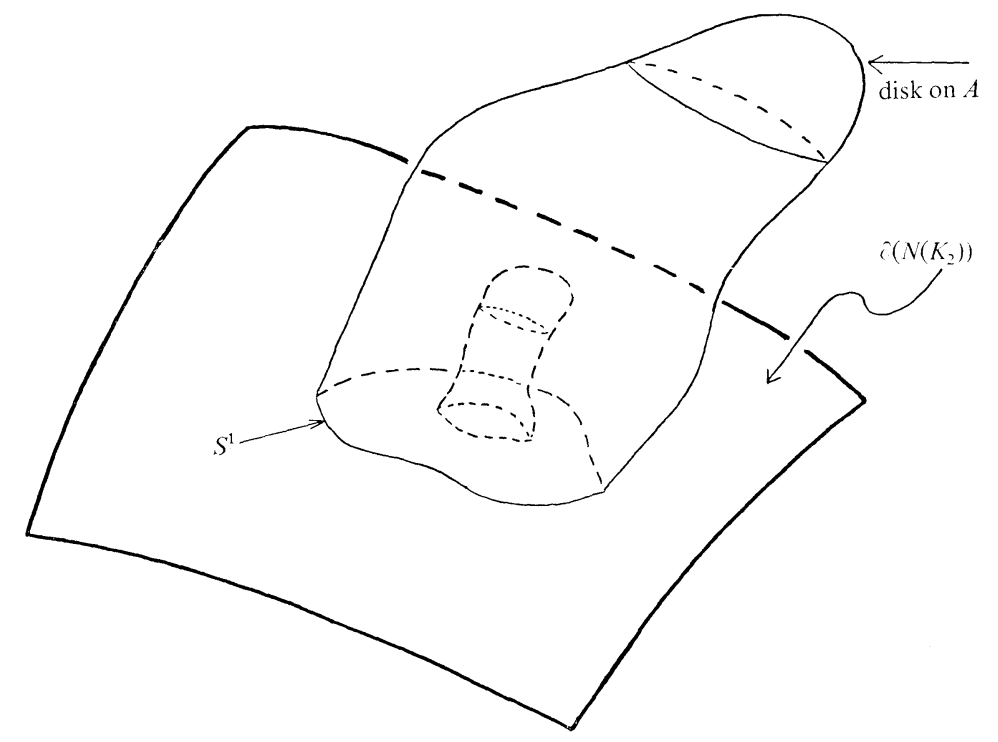

FIGURe 11

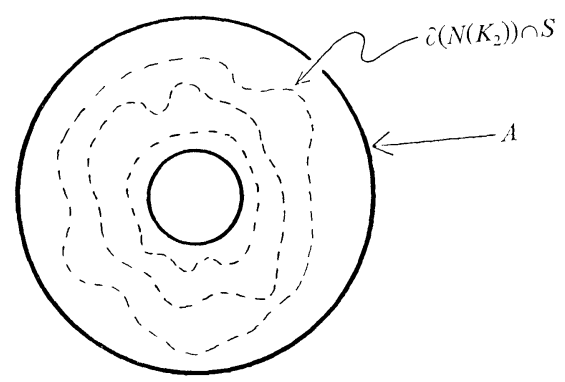

Figure 12 
After repeating this procedure we have that all circles of intersection on $A$ are parallel to the boundary of $A$. See Fig. 12. If any such circle existed, however, by considering an innermost one we would get wrapping number $K_{1}=1$, contradicting the hypothesis. By the initial remark, we have completed the proof.

5. Prime knots in $S^{1} \times B^{2}$. In order to apply Theorem 4.2 we need examples of prime knots in $S^{1} \times B^{2}$. In this section we will provide a large number of such examples. Many of these examples were known to Schubert [6]. In what follows we will consider $S^{1} \times B^{2}$ embedded in $S^{3}$ in a standard way. Hence any knot $K$ in $S^{1} \times B^{2}$ gives rise to a knot $K^{*}$ in $S^{3}$. Basic examples derive from the following result.

Proposition 5.1. If $K$ is a knot in $S^{1} \times B^{2}$ and $K^{*}$ is trivial in $S^{3}$, then $K$ is prime in $S^{1} \times B^{2}$.

Proof. Let $S$ be a splitting- $S^{2}$ for $K$ in $S^{1} \times B^{2}$. It is also a splitting- $S^{2}$ for $K^{*} . K^{*}$ is trivial implies that for any ball bounded by $S, B^{3},\left(B^{3}, B^{3} \cap K^{*}\right)$ is trivial, so $\left(B^{3}, B^{3} \cap K\right)$ is also trivial.

CoRollary 5.2. Doubled knots are prime.

Proof. Doubled knots are obtained by taking the $K_{1}$ cable about some knot, where $K_{1}$ is pictured in Fig. 13. Note that $K_{1}^{*}$ is trivial. (This result also follows from a genus argument.)

COROLlaRY 5.3. Every knot is concordant to a prime knot.

Proof. Apply the concordance used in proving Theorem 3.2. The new knot is the $K_{2}$ cable about some knot, with $K_{2}$ as in Fig. 13. Note that $K_{2}^{*}$ is trivial.
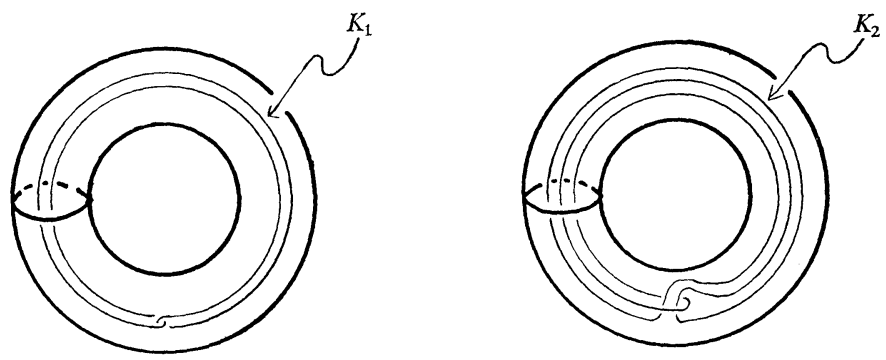

FIGURE 13

REMARK. The only prime knot in $S^{1} \times B^{2}$ with wrapping number 
0 is trivial, the only prime knot in $S^{1} \times B^{2}$ with wrapping number 1 is the core. Both of these results are straightforward. Hence to see that $K_{1}$ above does not have wrapping number 0 and that $K_{2}$ does not have wrapping number 1 , note that -1 surgery on each gives a nontrivial knot complement. See Figs. 4 and 7 . This is not the case for either the core of $S^{1} \times B^{2}$ or a trivial circle.

LEMMA 5.4. Let $K$ be a knot in $S^{1} \times B^{2}$ with wrapping number $=$ n. Let $D$ be a disk in $S^{1} \times B^{2}$ with $\partial D=$ meridian of $S^{1} \times B^{2}$ and $D \cap K=n$ points. If $K$ is not prime, there is a splitting $-S^{2}, S$, for $K$, with $S=\partial B^{3},\left(B^{3}, B^{3} \cap K\right)$ nontrivial, and $S \cap D=\varnothing$.

Proof. See Fig. 14. Denote by $\underline{A}\{S$-neighborhoods of $K \cap S\}$. We will use an innermost circle argument to eliminate circles of intersection.
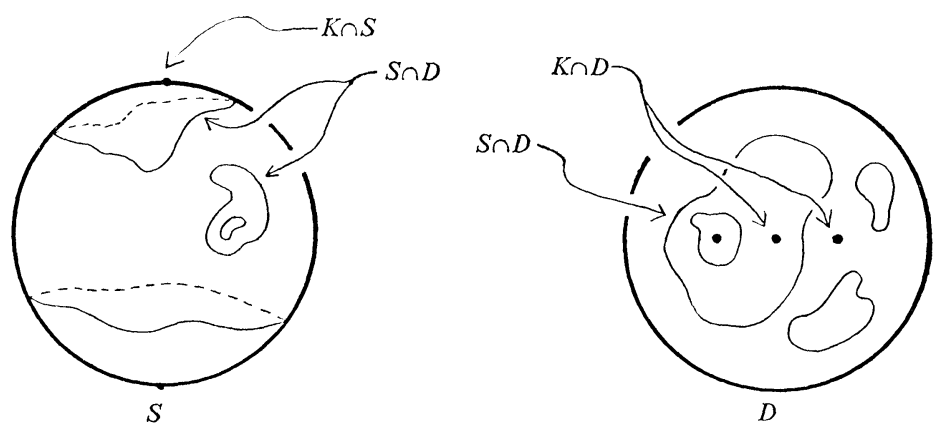

FiguRe 14

If any circle of intersection is null homologous on $A$, take an innermost such circle, $S^{1}$. If $S^{1}$ is null homologous on $D-K$, we can eliminate that circle of intersection using an isotopy. See Fig. 15. If $S^{1}$ is not null homologous on $D-K$, by replacing the disk it bounds on $D$ with the one it bounds on $S$, we would get a
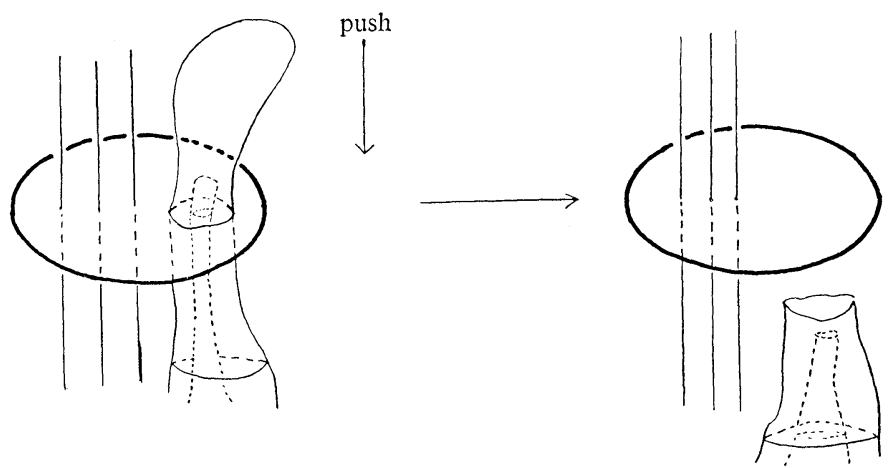
meridinal disk meeting $K$ in fewer than $n$ points, contradicting the fact that wrapping number $K=n$. Hence, after an isotopy, all circles of intersection can be assumed to be parallel to $\partial A$.

Now consider an innermost circle of intersection on $A$, that is one closest to the boundary, $S^{1}$. $S^{1}$ links $D \cap K$ algebraically once on $D$. But it also must link it geometrically once. If it linked more than once we could remove the disk on $K$ bounded by $S^{1}$ with the one it bounds on $S$. This would again contradict the wrapping number of $K$.

The disk bounded by $S^{1}$ on $D$ along with the one bounded by $S^{1}$ on $S$ form a splitting $-S^{2}, S^{\prime}$, for $K$, which after a small isotopy misses $D$. It bounds a 3 -ball, $B^{3}$, missing $D$. If $\left(B^{3}, B^{3} \cap K\right)$ is nontrivial we are done. If it is trivial we can use $B$ to isotop $S$ (fixing $K$ ) to eliminate at least one circle of intersection. After repeated application of this procedure we get the desired result. Fig. 16 illustrates this procedure.

REMARK. This procedure can be repeated to eliminate intersections with any disjoint, finite collection of such disks.
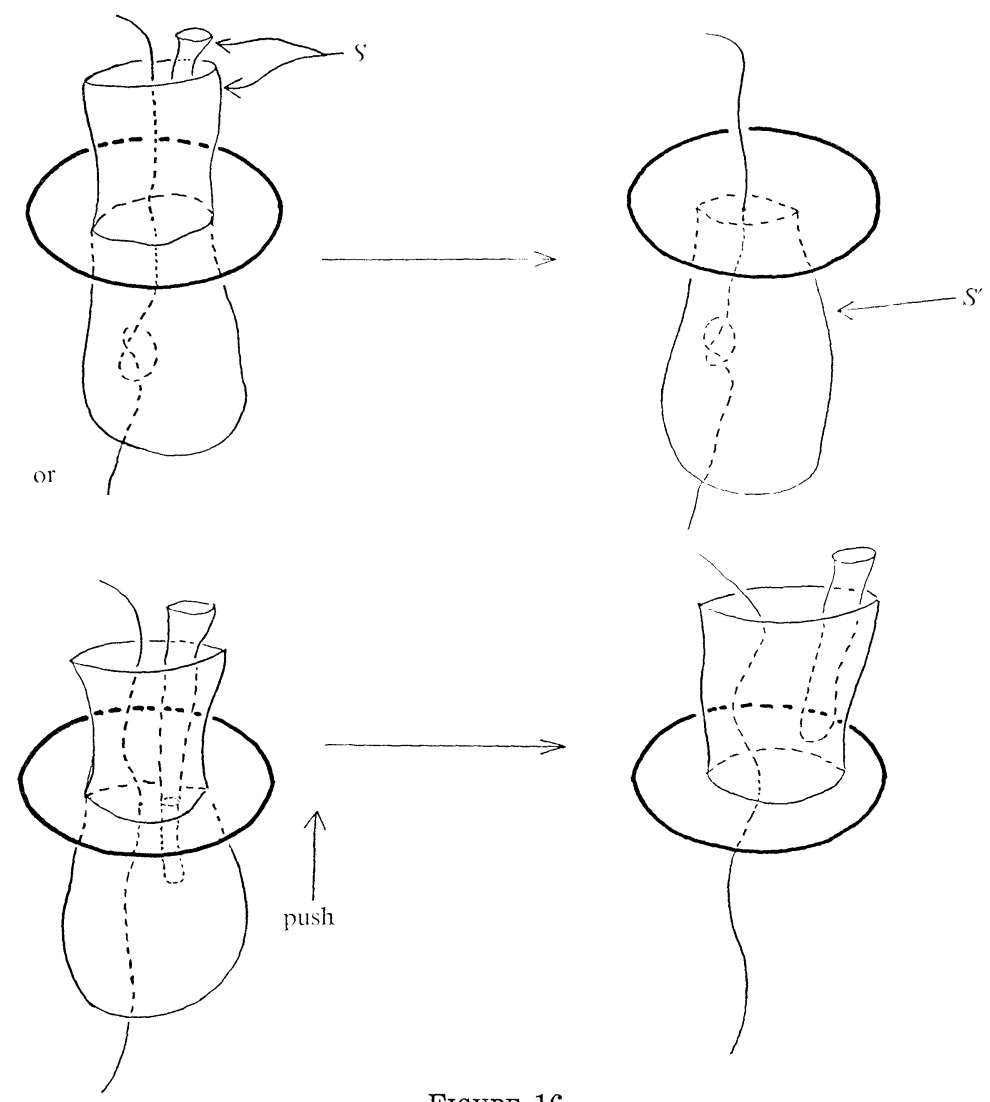

Figure 16 
Let $K$ denote a knot in $S^{1} \times B^{2}$. Cutting $S^{1} \times B^{2}$ along a meridinal disk gives a $B^{3}$ with a collection of embedded arcs. Any such collection of embeddings will be called prime if for any splitting$S^{2}$, for an arc, $S$ bounds a 3 -ball, $B^{3}$, with $\left(B^{3}, B^{3} \cap\right.$ arc $)$ trivial. See Fig. 17.
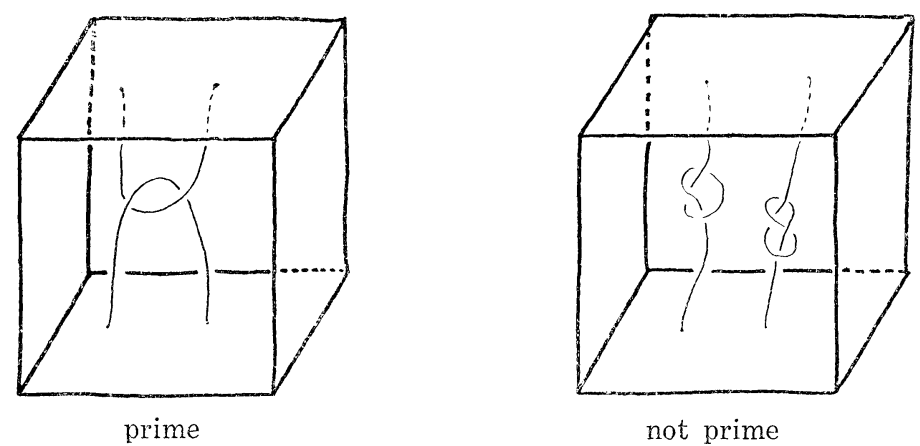

FIGURE 17

Proposition 5.5. Let $K$ be a knot in $S^{1} \times B^{2}$ and $\left\{D_{i}\right\}$ be a collection of disjoint disks with $\partial D_{i}=$ meridian, and each $D_{i}$ intersecting $K$ in a minimum number of points. If each $B^{3}$ obtained by cutting $S^{1} \times B^{2}$ along the $D_{\imath}$ 's contains a prime collection of ares then $K$ is prime, and conversely.

Proof. If $K$ is not prime, by Lemma 5.4 and the remark following it, there is a splitting- $S^{2}, S$, for $K$, with $S \cap D=\varnothing$ for all $i$, and with $S=\partial B^{3}$, where $\left(B^{3}, B^{3} \cap K\right)$ is nontrivial. Cutting $S^{1} \times B^{2}$ along the $D_{\imath}$ 's does not effect $S, B^{3}$, or $B^{3} \cap K$, so one of the collection of ares is not prime. The converse is immediate.

Schubert's results [6] that if cutting along a single disk yeilds a $B^{3}$ with each arc trivial, then the $K$ cable about any knot is prime follows immediately. For example:

Corollary 5.6. Any braid in $S^{1} \times B^{2}$ is prime.

Corollary 5.7. Any Mazur knot in $S^{1} \times B^{2}$ is prime. See Fig. 18.

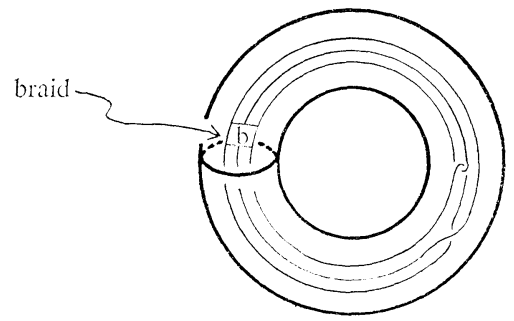

FIGURE 18 
As a final example, the knot in Fig. 19 is prime. If it were not prime we would have the collection of arcs in Fig. 20a not prime, in which case those in Fig. $20 \mathrm{~b}$ would not be prime. But they are prime as the knot in Fig. 20c is prime, which follows from Proposition 5.1.

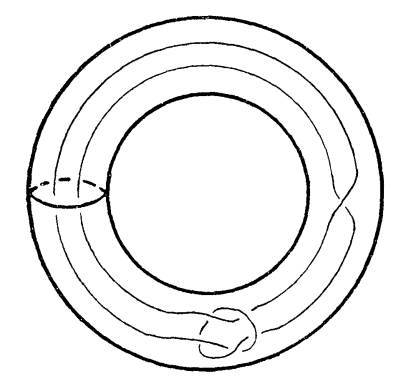

FIGURe 19

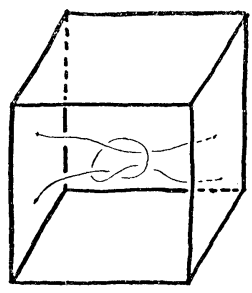

20a.

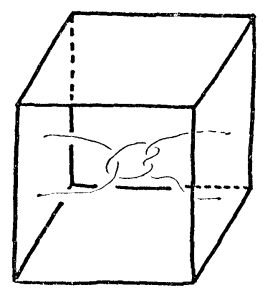

$20 \mathrm{~b}$.

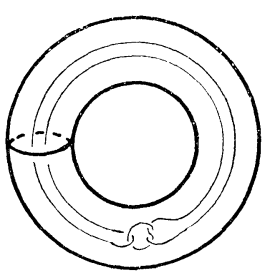

$20 \mathrm{c}$.

FIGURE 20

\section{REFERENCES}

1. C. McA. Gordon, Knots, homology spheres, and contractible 4-manifolds, Topology, 14 (1975), 151-172.

2. - Problems in knot theory, Lecture Notes in Math., 685, Knot Theory Proceeding (Plans-sur-Bex), Springer Verlag, (1978), 309-311.

3. R. Kirby and W. B. R. Lickorish, Prime knots and concordances, Proc. Camb. Phil. Soc., 86 (1979), 437-441,

4. W. B. R. Lickorish, A representation of orientable, combinatorial 3-manifolds, Ann. of Math., 76 (1962), 531-538.

5. D. Rolfsen, Knots and Links, Publish or Perish, Inc., Berkeley, Calif., 1976.

6. H. Schubert, Knoten und vollringe, Acta Math., 90 (1953), 131-286.

Received April 2, 1979.

RICE UNIVERSITY

HOUston, TX 77001 


\section{PACIFIC JOURNAL OF MATHEMATICS}

\section{EDITORS}

DONALD BABBITT (Managing Editor)

University of Galifornia

Los Angeles, California 90024

Hugo RossI

University of Utah

Salt Lake City, UT 84112

C. C. MOORE AND ANDREW OGG

University of California

Berkeley, CA 94720

\section{J. DugundjI}

Department of Mathematics University of Southern California

Los Angeles, California 90007

R. Finn AND J. Milgram

Stanford University

Stanford, California 94305

\section{ASSOCIATE EDITORS}

R. ARENS

E. F. BECKENBACH

B. H. NeUmanN

F. WOLF

K. YosHIDA

\section{SUPPORTING INSTITUTIONS}

UNIVERSITY OF ARIZONA

UNIVERSITY OF BRITISH COLUMBIA

CALIFORNIA INSTITUTE OF TECHNOLOGY

UNIVERSITY OF CALIFORNIA

MONTANA STATE UNIVERSITY

UNIVERSITY OF NEVADA, RENO

NEW MEXICO STATE UNIVERSITY

OREGON STATE UNIVERSITY
UNIVERSITY OF OREGON

UNIVERSITY OF SOUTHERN CALIFONIA

STANFORD UNIVERSITY

UNIVERSITY OF HAWAII

UNIVERSITY OF TOKYO

UNIVERSITY OF UTAH

WASHINGTON STATE UNIVERSITY

UNIVERSITY OF WASHINGTON 


\section{Pacific Journal of Mathematics}

\section{Vol. 94, No. 1 \\ May, 1981}

Willy Brandal, Conditions for being an FGC domain $\ldots \ldots \ldots \ldots \ldots \ldots \ldots$

Allan Calder and Frank Williams, Incompressibility of maps and the

homotopy invariance of Čech cohomology $\ldots \ldots \ldots \ldots \ldots \ldots \ldots \ldots \ldots$

Jacques Chaumat, Quelques propriétés du couple d'espaces vectoriels

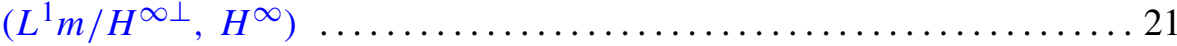

Manfred Droste and Rüdiger Göbel, Products of conjugate permutations . . 47

Jean Esterle, Rates of decrease of sequences of powers in commutative

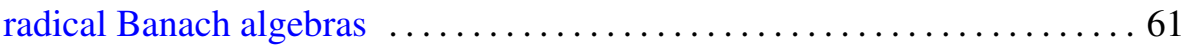

Allan Fryant, Ultraspherical expansions and pseudo analytic functions . . . 83

John Hannah, Homogenization of regular rings of bounded index. II . . . . . 107

Shigeru Haruki, On the theorem of S. Kakutani-M. Nagumo and J. L. Walsh

for the mean value property of harmonic and complex polynomials $\ldots .113$

Hugh M. Hilden, Representations of homology 3-spheres $\ldots \ldots \ldots \ldots \ldots 125$

Craig Huneke, A characterization of locally Macaulay completions . . . . . 131

Takesi Isiwata, Closed ultrafilters and realcompactness ................. 139

Joseph Weston Kitchen, Jr. and David A. Robbins, Tensor products of

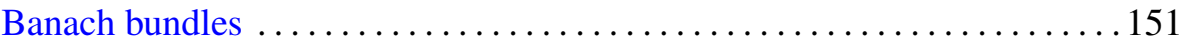

Allan J. Kroopnick, Note on bounded $L^{p}$-solutions of a generalized Liénard

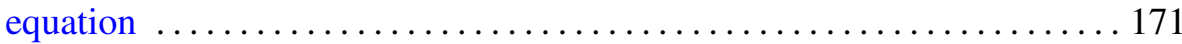

Ajay Kumar and Ajit Kaur Chilana, Spectral synthesis in products and quotients of hypergroups

Charles Livingston, Homology cobordisms of 3-manifolds, knot

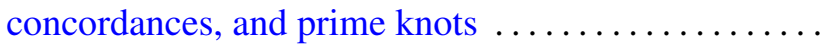

Hans Opolka, Projective representations of finite groups in cyclotomic fields

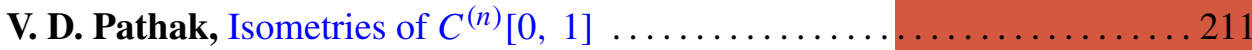

Mark Allan Pinsky, On the spectrum of Cartan-Hadamard manifolds . . . . . 2223

Judith Roitman, The number of automorphisms of an atomic Boolean

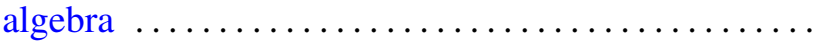

Kai Wang, Locally smooth torus group actions on integral cohomology complex projective spaces 